\title{
Evaluación de la licenciatura en Administración y Gestión de Pequeñas y Medianas Empresas por CIEES: gestión de innovación en la UPVE
}

\author{
Evaluation of the Bachelor's Degree Administration and Management of \\ Small and Medium Enterprises by CIEES: Innovation Management at UPVE \\ Avaliação da licenciatura em Administração e Gestão de Pequenas e \\ Médias Empresas pelo CIEES: gestão da inovação na UPVE
}

Imelda Zayas Barreras Universidad Politécnica del Valle del Évora, México

imelda.zayas@upve.edu.mx https://orcid.org/0000-0002-5643-5711

\section{Resumen}

El objetivo principal de la presente investigación fue documentar el proceso seguido por la Universidad Politécnica del Valle del Évora (UPVE) para conseguir la acreditación de la licenciatura en Administración y Gestión de Pequeñas y Medianas Empresas. Para cumplir con dicho objetivo se empleó el método cualitativo, el cual permitió realizar descripciones detalladas de eventos. Luego de cumplir con el proceso indicado, se puede indicar que el Comité Interinstitucional para la Evaluación de Educación Superior (CIEES), a través de comité del área de ciencias administrativas, dictaminó la acreditación del referido programa, con algunas observaciones de mejora continua, las cuales se están solventando progresivamente para el beneficio de los aspirantes, estudiantes y egresados. En conclusión, se puede señalar que existe escasa literatura sobre el tema estudiado, en especial sobre los modelos o esquemas de evaluación empleados para la acreditación de un programa, de ahí que se considere que la información suministrada en este documento puede servir como apoyo para otras universidades que decidan evaluar y acreditar un programa educativo. 


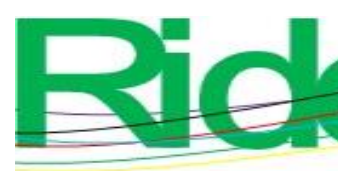

Revista Iberoamericana para la Investigación y el Desarrollo Educativo ISSN $2007-7467$

Palabras Clave: Programas educativos, calidad, acreditación, mejora continua, evaluación externa.

\section{Abstract}

The main objective of this research was to document the process followed by the Évora Valley Polytechnic University (UPVE) to obtain the accreditation of the degree in Administration and Management of Small and Medium Enterprises. To meet this objective, the qualitative method was used, which allowed detailed descriptions of events. After complying with the indicated process, it can be indicated that the Interinstitutional Committee for the Evaluation of Higher Education (CIEES), through a committee of the administrative sciences area, ruled the accreditation of the referred program, with some observations of continuous improvement, the which are progressively being resolved for the benefit of applicants, students and graduates. In conclusion, it can be noted that there is little literature on the subject studied, especially on the evaluation models or schemes used for the accreditation of a program, hence it is considered that the information provided in this document may serve as support for other universities that decide to evaluate and accredit an educational program.

Keywords: Educational programs, quality, accreditation, continuous improvement, external evaluation.

\section{Resumo}

O principal objetivo desta pesquisa foi documentar o processo seguido pela Universidade Politécnica do Vale de Évora (UPVE) para a obtenção da acreditação do curso de Administração e Gestão de Pequenas e Médias Empresas. Para atender a esse objetivo, foi utilizado o método qualitativo, que permitiu a descrição detalhada dos eventos. Cumprido o processo indicado, pode-se indicar que a Comissão Interinstitucional de Avaliação do Ensino Superior (CIEES), através de uma comissão da área das ciências administrativas, regulamentou o credenciamento do referido programa, com algumas observações de melhoria contínua, o que estão sendo progressivamente resolvidos em benefício dos candidatos, alunos e graduados. Concluindo, nota-se que existe pouca literatura sobre o tema estudado, principalmente sobre os modelos ou esquemas de avaliação utilizados para o credenciamento de um programa, portanto considera-se que as informações prestadas neste 


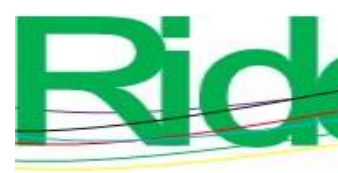

Revista Iberoamericana para la Investigación y el Desarrollo Educativo ISSN 2007 - 7467

documento podem servir de suporte para outros universidades que decidem avaliar e credenciar um programa educacional.

Palavras-chave: Programas educacionais, qualidade, acreditação, melhoria contínua, avaliação externa.

Fecha Recepción: Junio 2020

Fecha Aceptación: Noviembre 2020

\section{Introducción}

El principal objetivo de la evaluación y acreditación de los programas universitarios es mejorar la calidad de la educación superior a través de recomendaciones para la optimización de las actividades. Esto se logra, evidentemente, revisando con detalle la manera en que se encuentra operando el programa educativo para detectar fortalezas, debilidades, amenazas y oportunidades. En el caso de México, por ejemplo, se han creado organismos que tienen como prioridad evaluar directamente ese tipo de programas. Al respecto, Acosta Ochoa (2014) señala:

En la actualidad, existen diversos organismos evaluadores especializados en las diversas disciplinas, pero son dos las instancias que aglutinan estas tareas: a) los Comités Interinstitucionales para la Evaluación de la Educación Superior (CIEES), surgidos en 1991, que concentran su actividad en el diagnóstico y la evaluación de los programas educativos con base en la asignación del nivel 1 (programas de calidad o pre-acreditados); b) el Consejo para la Acreditación de la Educación Superior (COPAES), creado en el año 2000 y que constituye el único organismo avalado por la Secretaría de Educación Pública (SEP) para otorgar la acreditación de los programas, o por decirlo de algún modo, funge como el "acreditador de los acreditadores (p. 1 ) .

Al evaluar los programas educativos, los dos organismos referidos emiten un veredicto en el cual indican el nivel de calidad, lo cual sirve como un elemento de prestigio que puede ser tomado en cuenta por los estudiantes de bachillerato al momento de decidirse por alguna opción universitaria. Aun así, desde los sectores empresarial y social se han emitido críticas en cuanto a la idoneidad de los programas educativos, lo cual ha obligado a las instituciones a rendir cuentas en torno a sus actividades académicas. 


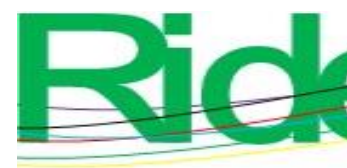

Revista Iberoamericana para la Investigación y el Desarrollo Educativo ISSN $2007-7467$

Las duras críticas a las que se ven sometidas las instituciones de educación superior (IES) en cuanto a las respuestas esperadas por los sectores productivo y social colocan a la evaluación, la certificación y la acreditación como los mecanismos para el aseguramiento de la calidad educativa, de regulación y autorregulación, que encuentran su máxima justificación en lo que se conoce como "la rendición de cuentas" y como las estrategias que por excelencia deberían permitir las transformaciones necesarias y el cumplimiento de las expectativas que están puestas en la educación superior (Hernández Mondragón, 2006, p. 53 ).

Los programas de educación superior en México se han incrementado en los últimos veinte años debido al surgimiento de universidades públicas y privadas que procuran ofertar currículos que atiendan los requerimientos de la población estudiantil. Sin embargo, para conseguir la acreditación y la certificación, esos programas deben ser evaluados por organismos externos avalados por la Secretaría de Educación Pública

La evaluación y acreditación de la educación superior en México se realiza actualmente por un amplio conjunto de organismos e instancias especializados. Este conjunto ha construido, a la fecha, un vasto sistema de marcos de referencia, criterios, indicadores, estándares, instrumentos de medición y estrategias de promoción que tienen como objetivo fundamental contribuir a la mejora continua y al aseguramiento de la calidad de las instituciones de educación superior, y con ello al logro de la equidad educativa. Este conjunto de organismos e instancias especializadas cubre los ámbitos de evaluación de alumnos (Instituciones de Educación Superior IES, Centro Nacional de Evaluación para la Educación Superior CENEVAL, egresados (IES, CENEVAL), personal académico (IES, Sistema Nacional de Investigadores SNI), programas educativos de los niveles de técnico superior universitario, profesional asociado y licenciatura (IES, Comités Interinstitucionales para la Evaluación de la Educación Superior CIEES, organismos acreditadores reconocidos por el Consejo para la Acreditación de la Educación Superior COPAES, programas educativos de posgrado (IES, CIEES, Padrón Nacional de posgrado SEP-CONACYT), e instituciones (IES, 


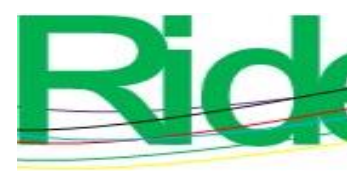

Revista Iberoamericana para la
Investigación y el Desarrollo Educativo
ISSN $2007-7467$

Federación de Instituciones Mexicanas Particulares de Educación Superior FIMPES) (Rubio Oca, 2007, p. 36).

Existen diversos organismos encargados de valorar cada una de las áreas que componen el sistema educativo superior, es decir, alumnos, egresados, docentes, programas educativos de pregrado y posgrado, etc. En el caso del personal académico, por ejemplo, existe el Programa para el Desarrollo Profesional Docente (Prodep), que evalúa a los docentes según su producción científica y tecnológica, entre otros factores.

Asimismo, en cada uno de los estados de la república mexicana funciona un organismo dependiente del Consejo Nacional de Ciencia y Tecnología (Conacyt), que también revisa la producción científica y tecnológica, así como la divulgación, el desarrollo humano y las distinciones a través de convocatorias. En el caso de Sinaloa, el Instituto Nacional de Propiedad Industrial (INAPI) promueve la convocatoria del Sistema Sinaloense de Investigadores y Tecnólogos (SSIT) para categorizar la producción de los docentes según tres categorías: investigador o tecnólogo asistente, investigador tecnólogo e investigador honorífico.

Ahora bien, el proceso de acreditación de los programas educativos se enfoca en todas las actividades administrativas y de docencia de la universidad con la finalidad de proporcionar información real y fidedigna sobre la oferta educativa que se brinda a la sociedad.

La acreditación universitaria es el resultado de un proceso de evaluación y seguimiento sistemático y voluntario del cumplimiento de las funciones sustantivas de una institución de educación superior (IES), que permite obtener información fidedigna y objetiva sobre la calidad de las instituciones. Permite certificar, ante la sociedad, la calidad de los recursos humanos formados y de los diferentes procesos que tienen lugar. Es el reconocimiento formal y público otorgado a una institución académica en virtud del grado en que ha logrado avances significativos en sus carreras o programas en el cumplimiento de su misión y objetivos declarados, y satisface un conjunto de criterios, indicadores y estándares de pertinencia y calidad. El propósito central de la acreditación es promover y estimular el continuo mejoramiento y determinar si una institución académica posee calidad a nivel general o respecto de uno o más programas educativos, si es capaz de demostrar que 


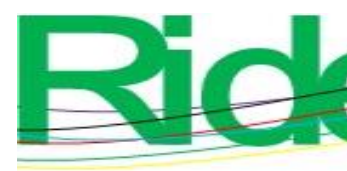

Revista Iberoamericana para la Investigación y el Desarrollo Educativo ISSN $2007-7467$

progresa de manera continua y sistemática, con el empleo de estrategias, procedimientos y recursos adecuados para el logro de su misión y sus objetivos, cumpliendo razonablemente con los criterios y normas de calidad establecidos (Rodríguez Pérez, 2014, p. 3).

La acreditación de un programa educativo, en primera instancia, supone un reconocimiento formal y público ante la sociedad, lo cual sirve de referencia para los aspirantes que desean cursar determinada carrera. Sin embargo, como lo plantean Castillo Marrufo, Aragón García y Hernández Jaime (2014), ese proceso implica diversas actividades que procuran un mejoramiento constante de la educación.

La evaluación y la acreditación no son fines en sí mismos, sino medios para promover el mejoramiento de la educación superior. Es usual asociar ambas actividades con el mejoramiento de la calidad, la generación de información para la toma de decisiones y la garantía pública de la calidad de las instituciones y de los programas académicos que ofrecen.

La acreditación es un proceso que involucra comparar programas académicos o instituciones con estándares previamente establecidos por un organismo facultado para otorgar la correspondiente acreditación. El proceso es útil para asegurar y desarrollar la calidad de la educación ofrecida por las IES. Dicho proceso generalmente consiste de fases como la autoevaluación, la evaluación externa y una decisión de acreditación basada en los estándares. En términos generales, mientras que la evaluación tiene propósitos de diagnóstico y de formulación de recomendaciones encaminadas a elevar la calidad de las instituciones y de sus programas académicos, la acreditación compara programas académicos o IES con los estándares establecidos por organismos externos, los cuales tienen facultades para otorgar la acreditación. La diferencia fundamental entre evaluación y acreditación radica en el agente que evalúa o acredita. Mientras que el evaluador puede ser interno o externo, el acreditador es necesariamente externo (p. 2).

Por lo antes expuesto, es importante señalar que la evaluación y acreditación de los programas educativos es una tarea completamente voluntaria por parte de las instituciones que quieran obtener un reconocimiento externo de la calidad de su quehacer cotidiano en la educación. 


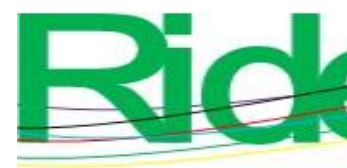

Revista Iberoamericana para la
Investigación y el Desarrollo Educativo
ISSN $2007-7467$

En cuanto a su definición, la acreditación es entendida como un proceso voluntario por medio del cual una institución educativa se somete a la opinión de un organismo externo, con la intención de obtener un reconocimiento público de la calidad de su quehacer educativo, pudiéndose aplicar tanto a las instituciones como a los programas educativos. Por lo regular, el proceso a seguir inicia con la autoevaluación institucional, seguido por la evaluación y opinión que realizan pares académicos externos a la institución educativa, y se finaliza con el informe de resultados sobre la acreditación de la calidad educativa (Martínez Iñiguez, Tobón y Romero Sandoval, 2017, p. 80).

La evaluación se realiza por organismos externos a la institución para brindar garantía de trasparencia y calidad, lo cual se logra a través del dictamen generado en el que se indican los aspectos que se pueden mejorar. Es importante señalar que acreditar un programa educativo sirve para informar a la sociedad sobre cuáles universidades imparten carreras certificadas y de calidad.

A las instituciones educativas, la acreditación les trae como beneficio la mejora de los programas académicos al atender las recomendaciones de los organismos acreditadores; el reconocimiento público como instituciones de prestigio académico; y el acceso a programas de apoyo institucionales que contribuyan a la mejora integral de su capital humano y equipamiento e infraestructura, entre otros; sin embargo la mejora más importante es que la institución se organiza para cumplir sus objetivos estratégicos con una orientación hacia la mejora continua, que los estudiantes aprendan y tener capacidad de respuesta a las necesidades de la sociedad. Por lo que se refiere a los empleadores, les permite participar y tener información respecto a la calidad de los programas para mantener relaciones que permitan una vinculación adecuada — educación continua y proyectos conjuntos - y recibir en sus espacios laborales a profesionistas competentes (Copaes, 2016, p. 7).

Es importante destacar que al evaluar un programa de estudios se involucran a todos los participantes y se generan algunas recomendaciones en torno a estrategias que se pueden implementar para mejorar la planeación y el desarrollo de competencias. 


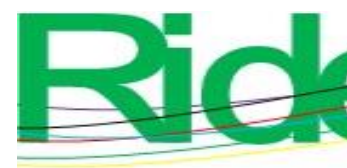

Revista Iberoamericana para la
Investigación y el Desarrollo Educativo ISSN $2007-7467$

La evaluación en educación superior se basa principalmente en la implementación de estrategias, para mejorar la planeación educativa propuesta, no dejando de lado la importancia de logro de los objetivos propuestos para alcanzar el currículo y al mismo tiempo desarrollar las competencias de formas válidas y fiables, pues esto implica grandes cambios de enseñanza por la demanda de las nuevas necesidades sociales. Los estudiantes se encuentran en situaciones de mayor complejidad, es decir, que logren una competencia científica y técnica, adquieran actitudes, mejoren sus valores y habilidades, creando en ellos una persona con amplio criterio para poder decidir por sí mismos sobre las oportunidades que se les presenten y sea mejor de acuerdo al nivel profesional (Rosales Almazán, 2019, p. 2).

La evaluación de la educación superior, en otras palabras, tiene sus grandes ventajas para los estudiantes porque se promueve la comparación y la competencia con otros programas educativos. Esto, a largo plazo, favorece a los alumnos, quienes deben desarrollar y actualizar sus habilidades, competencias, valores, aptitudes y actitudes.

Ahora bien, a pesar de todos los beneficios mencionados, cabe señalar que en algunos casos durante el proceso de evaluación se presentan limitaciones debido al reducido número de participantes de la institución que realizan la autoevaluación. Es decir, en ocasiones se requieren documentos a los cuales solo puede acceder el personal administrativo, los cuales pueden no tener la disponibilidad de colaborar. Además, los docentes pueden ver el proceso como una simple evaluación, de modo que no le dan la importancia necesaria. Los siguientes son algunos de los problemas a los cuales se debe enfrentar el proceso de acreditación:

- La simulación en los procesos de acreditación.

- El incremento del aparato burocrático dentro de las IES.

- La poca participación de los miembros de la comunidad educativa en los procesos de acreditación.

- La falta de credibilidad en los organismos externos.

- La falta de aplicación del currículo por competencias.

- La ausencia de impacto respecto a los fines de la universidad.

Por ende, es preciso estudiar estas problemáticas con el fin de implementar mejoras continuas en los procesos académicos que lleven a formar profesionales que estén en condiciones de afrontar los retos actuales y futuros 


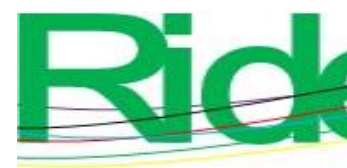

Revista Iberoamericana para la
Investigación y el Desarrollo Educativo
ISSN $2007-7467$

de la sociedad, la ciencia, la tecnología y las organizaciones, algo que casi no está ocurriendo en las universidades acreditadas (Martínez Iñiguez et al., 2017, p. 81).

En todas las instituciones educativas se presentan problemas al momento de tomar la decisión de evaluar o acreditar un programa educativo; por ejemplo, conseguir financiamiento, elegir al organismo evaluador, integrar al equipo colaborador y, sobre todo, concienciar a toda la comunidad universitaria de la importancia del proceso.

Aunado a esto, otros autores señalan que la evaluación de un programa educativo puede presentar algunas limitaciones evidenciadas por los estudiantes.

El sistema de evaluación y acreditación de la educación superior en México se ha complejizado a lo largo de los últimos treinta años. Si bien esto ha mejorado de forma importante la equidad y, en lo general las experiencias que acompañan a los estudiantes a lo largo de su carrera, en opinión de los alumnos existen diversas limitaciones que no permiten sedimentar los esfuerzos que se han llevado a cabo para generar procesos de aseguramiento de la calidad de los programas educativos, y los diversos elementos que los constituyen académica y administrativamente (Buendía Espinosa, Sampedro Hernández y Acosta Ochoa, 2013, p. 66).

Al evaluar un programa educativo, algunos organismos (por ejemplo el CIEES) al realizar la evaluación diagnóstica no involucran a los estudiantes y a algunos docentes, los cuales solo son informados del proceso.

Explicado lo anterior, la Universidad Politécnica del Valle del Évora ha decidido realizar la evaluación del programa educativo licenciatura en Administración y Gestión de Pequeñas y Medianas Empresas, a través de Comités Interinstitucionales para la Evaluación de la Educación Superior, organismo externo que se enfoca en la evaluación diagnóstica.

Los CIEES son el organismo que inició la evaluación externa de las instituciones de educación superior (IES) en México. Estos comités elaboraron y pusieron a disposición de la comunidad académica los primeros instrumentos con ese propósito, mismos que se han modificado, adecuado y actualizado para responder a las necesidades y los retos de nuestra época. Con el objetivo de que las IES de México aceptaran la evaluación externa, a partir de 1991 los CIEES propusieron a las IES llevar a cabo evaluaciones 


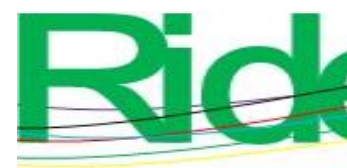

Revista Iberoamericana para la
Investigación y el Desarrollo Educativo
ISSN $2007-7467$

diagnósticas. Esto contribuyó significativamente a desarrollar entre las instituciones la cultura de la evaluación académica externa y fue la base sobre la que, poco a poco, se impulsó la creación y consolidación de los organismos acreditadores de la educación superior en México (CIEES, 2018, p. 5).

Este organismo ha impulsado el proceso de evaluación en instituciones de educación superior con la finalidad de atender una necesidad de la sociedad en general: conocer los programas de estudios de calidad sometidos a un proceso en el cual se indican fortalezas y debilidades que se deben atender.

$\mathrm{Al}$ respecto, es necesario explicar que la evaluación es efectuada por dos especialistas designados por el CIEES. Estas personas se encargan de evaluar el programa y de establecer comunicación directa con el personal para conocer a la IES y a las condiciones con las que opera.

Realizado un análisis de la evaluación diagnóstica, los programas de estudios de nivel superior deben ser actualizados en periodos cortos; además, se fomenta la movilidad de los estudiantes y docentes, se verifica la infraestructura existente y, si es necesario, se sugieren modificaciones en torno a los espacios para los estudiantes y para el personal administrativo y docente.

A partir de la descripción y comparación de los modelos de evaluación y acreditación institucional de organismos y programas, se discute el concepto de calidad que les subyace, y da cuenta de los efectos producidos. En un balance, reconoce como efectos positivos la actualización de los planes de estudio, la movilidad estudiantil, la renovación de la infraestructura, la incorporación de tecnología, la colaboración interinstitucional y los sistemas de gestión para el manejo de recursos. En contraparte, identifica que dichos modelos propician la simulación institucional, el cumplimiento de criterios e indicadores para mejorar la imagen de la institución, y que se obtengan recursos y beneficios fiscales que no repercuten en modificaciones sustantivas del funcionamiento interno de las instituciones (Rueda Beltrán y García Salord, 2013, p. 9).

Por otra parte, las evaluaciones de los CIEES sirven para que las instituciones trabajen en la mejora continua del programa educativo y se encaminen a la búsqueda de recursos a través de proyectos. 


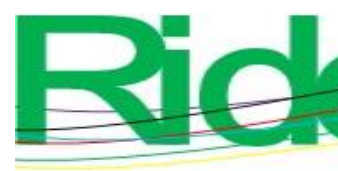

Revista Iberoamericana para la Investigación y el Desarrollo Educativo ISSN $2007-7467$

En cuanto a la metodología usada por los CIEES, vale indicar que incluye diversas variables, como registro del programa, estudiantes, docencia, segunda lengua utilizada, infraestructura, contenidos del programa y servicios de apoyo a los estudiantes, entre otras categorías de igual importancia.

Se debe señalar que tanto los comités de los CIEES como los organismos acreditadores de COPAES poseen marcos de referencia en los cuales establecen una serie de categorías e indicadores sobre los cuales se evalúan los Programas Educativos. Algunas categorías similares entre estas instancias son: personal académico, plan de estudios, métodos e instrumentos para evaluar el aprendizaje de los estudiantes, servicios de apoyo al estudiante, infraestructura, investigación, vinculación con los sectores de la sociedad, la normativa que regule la operación del programa, y la planeación y evaluación institucional.

Cabe destacar que todo PE tiene que realizar primero su proceso de evaluación diagnóstica ante un Comité de los CIEES, antes de que pueda solicitar la evaluación para la acreditación de un organismo reconocido por COPAES (Ramírez López, 2016, p. 129).

Una vez que la institución se registra para realizar la evaluación y acreditación del programa educativo en CIEES, se permite el acceso a una plataforma digital, donde los participantes del programa realizan un diagnóstico evaluado por el comité del área correspondiente. Luego de esta etapa, se puede solicitar la evaluación de acreditación del programa educativo ante otro organismo reconocido por Copaes, lo que constituye el paso previo a la valoración de un organismo más especializado en el área del programa. La intención de todo esto es ofrecer programas de calidad, así como equidad para que todos los aspirantes tengan la posibilidad de elegir determinada carrera.

La equidad educativa significa ofrecer igualdad de oportunidades educativas de buena calidad para todos; la evaluación y la acreditación de la educación superior adquieren, para el logro de este objetivo, una importancia estratégica, al identificar las desigualdades de calidad en los programas educativos y a la vez propiciar la mejora continua y el aseguramiento de la calidad de los mismos. Esta es la razón por la que, en las últimas dos décadas, la política orientada a fomentar la mejora de los insumos, procesos y resultados de la 


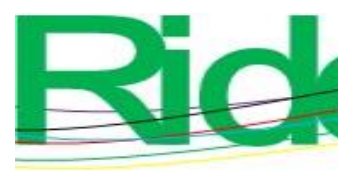

Revista Iberoamericana para la Investigación y el Desarrollo Educativo ISSN $2007-7467$

educación superior, ha ocupado un lugar destacado y de alta prioridad en el marco de las políticas educativas nacionales y estatales (De la Garza Aguilar, 2013, p. 34).

Por lo anterior, en los últimos años se ha promovido la valoración de los programas educativos por parte de organismos externos. Aunado a esto, se debe reconocer que la calidad de los programas educativos ha sido una preocupación de la Secretaría de Educación Pública en México, de ahí que desde los años noventa se haya trabajado en conseguir criterios apropiados para determinar su calidad.

El aseguramiento de la calidad ha sido una preocupación latente y legítima por diversos actores en el campo de la educación superior. Desde principios de la década de los noventa, la mayoría de los países han establecido mecanismos para velar por la calidad de ésta.

La acreditación surge como una figura utilizada para dar cuenta del proceso de aseguramiento de calidad basado en la evaluación y del "valor" o calidad de una institución o programa; considerándose la institucionalización más desarrollada de la idea de responsabilidad en la educación superior (Buendía Espinosa, 2011, p. 2).

Es importante destacar que al principio de estas iniciativas, solo las IES públicas se habían preocupado por someterse a esos procesos de evaluación; sin embargo, con el tiempo las IES privadas también se han ido interesando en ello debido a las recomendaciones que se pueden conseguir de la valoración de sus programas.

La evaluación en México se relacionó principalmente con la mejora de la calidad educativa y con la garantía de que las instituciones de educación superior fueran acreditadas respondiendo con ello a estándares preestablecidos.

Partiendo del principio de la mejora de la calidad educativa, la Federación de Instituciones Mexicanas Particulares de Educación Superior (FIMPES) contempló que la institución tiene una misión pertinente al contexto de la educación superior, y que cuenta con los recursos, programas y servicios suficientes para cumplir con ella, de ahí que las instituciones de educación superior particulares se sometieron también a estos ejercicios de evaluación 

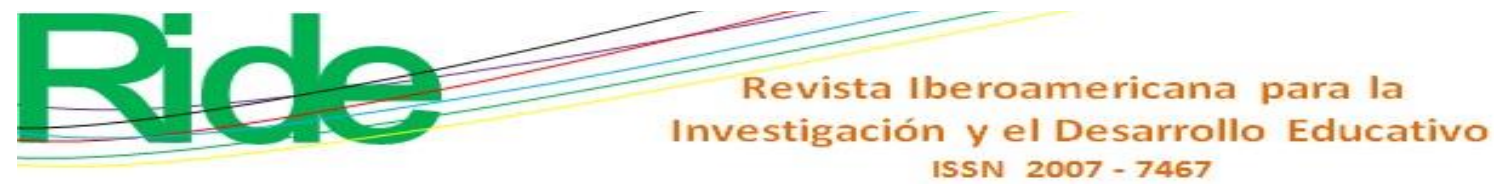

interna-externa para garantizar la pertinencia y calidad (Lara Valdés, 2013, p. $3)$.

En pocas palabras, es importante que las instituciones de educación superior desarrollen mecanismos que permitan conseguir la calidad de sus programas educativos, lo que ofrecerá a la institución un reconocimiento público para sus programas.

Si las IES logran estándares satisfactorios en el proceso de evaluación de calidad, entonces pueden certificarse y lograr la acreditación como la culminación de todo un proceso evaluativo, ya sea en programas académicos o a nivel institucional. Se entiende por certificación como el reconocimiento público y temporal del proceso enseñanza-aprendizaje y de la administración escolar gestionados con calidad, además de que las instituciones deben contar con la estructura requerida para la mejora permanente; y por acreditación se entiende al procedimiento que mediante un organismo especializado, avalado por el Consejo para la Acreditación de la Educación Superior (COPAES), reconoce formalmente que un programa educativo ha mostrado evidencias de calidad en su estructura, organización, funcionamiento, insumos, procesos de enseñanza, servicios, pertinencia social y resultados objetivos bajo criterios, indicadores y estándares de calidad comprobados por medio de las evaluaciones independientes e imparciales a las que se somete.

La acreditación de programas, por ejemplo, certifica la calidad de todos los elementos asociados al diseño curricular, recursos comprometidos y resultados del proceso enseñanza-aprendizaje. Es necesario mencionar que la acreditación tiene vigencia de cinco años, al término de este periodo, los programas son nuevamente evaluados (Arriaga Villanueva, 2013, p. 1).

Una institución de educación superior deberá tomar la decisión en la gestión que va a realizar para ofrecer sus programas de calidad, porque debe decidir si va a acreditar o certificar el programa educativo. La certificación es un reconocimiento temporal donde se señala que tanto el programa como sus procesos administrativos y estructura son de calidad; en cambio, la acreditación la emite Copaes, organismo externo que reconoce a través de evaluaciones imparciales la calidad tanto del programa como de todas sus funciones, insumos y procesos. 


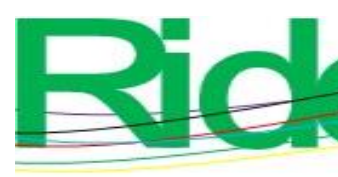

Revista Iberoamericana para la Investigación y el Desarrollo Educativo ISSN 2007 - 7467

Considerando que la calidad de la enseñanza superior se encuentra incluida dentro de las políticas públicas de nuestro país, se deberían incluir en la evaluación aspectos como las becas ofrecidas a los estudiantes, las investigaciones desarrolladas por docentes, estudiantes y personal administrativo, así como la infraestructura y el equipamiento, entre otros rubros de igual interés.

La calidad de la enseñanza superior es un concepto pluridimensional que debería comprender todas sus funciones y actividades: enseñanza y programas académicos, investigación y becas, personal, estudiantes, edificios, instalaciones, equipamiento y servicios a la comunidad y al mundo universitario. Una autoevaluación interna y un examen externo realizados con transparencia por expertos independientes, en lo posible especializados en lo internacional, son esenciales para garantizar la calidad.

Deberían crearse instancias nacionales independientes, y definirse normas comparativas de calidad, reconocidas en el ámbito internacional. Con miras a tener en cuenta la diversidad y evitar la uniformidad, debería prestarse la atención requerida a las particularidades de los contextos institucional, nacional y regional (González Vidaña, 2014, p. 5).

La autoevaluación promueve el crecimiento, especialmente cuando lo realiza un organismo externo; sin embargo, se debería de procurar la unificación de la normativa de evaluación usada por los diversos organismos; además, considerar la participación de reconocidas entidades internacionales para que existan criterios más precisos sobre la valoración de la calidad educativa. Este es un aspecto fundamental que no se debe soslayar, pues en medio de esta globalización las instituciones deben formar a profesionales competitivos. Por eso, no extraña que los gobiernos promuevan estrategias que permitan fomentar una educación de calidad que beneficie a la sociedad en general.

Desde hace dos décadas, con políticas públicas diversas, entre las que destaca la estrategia de aseguramiento de la calidad, la atención gubernamental de los gobiernos se ha venido concentrando en lograr que la educación que se imparta sea de buena calidad y asegurar los mínimos de calidad que deben tener las instituciones de educación superior (IES) públicas y privadas. A la fecha, es evidente que la evaluación y acreditación han sido factor de cambio y mejora en la educación superior del país. Sin embargo, es tiempo de realizar 


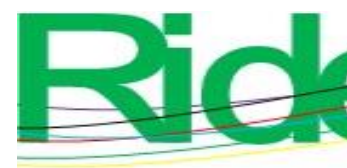
Revista Iberoamericana para la
Investigación y el Desarrollo Educativo
ISSN $2007-7467$

una profunda revisión de métodos y resultados de este proceso. El punto central a discusión es cómo mantener una estrecha correspondencia con la gran transformación que ha provocado el desarrollo tecnológico y la globalización en nuestro sistema educativo y nuestros mecanismos de aseguramiento de la calidad (Zenteno Trejo, Osorno Sánchez y López Portillo Tostado, 2017, p. 14).

En concordancia con esta idea, Corona Zapata (2014) hace referencia a la importancia de la evaluación de los programas educativos para asegurar la calidad de la oferta educativa de las IES.

Para que la evaluación y acreditación de programas educativos contribuya realmente al mejoramiento de la educación superior, es necesario reconocer que son igualmente necesarios otros tipos de evaluación y ponerlos en marcha; contar con reconocimiento de calidad no debería limitar a las instituciones de educación superior a seguir analizando desde distintas aristas, la calidad que dice ser ofertada en sus programas (Corona Zapata, 2014, p. 2).

Un programa educativo con calidad no debe de limitarse a un reconocimiento momentáneo, sino que debe de seguir analizando todas sus variables para brindar una mejora continua. En este sentido, los programas educativos a distancia también tienen que ser evaluados y acreditados para que se indique no solo su calidad, sino también la idoneidad de sus estrategias para tomar clases virtuales.

Evaluar la calidad educativa no es un proceso fácil o simple, y hasta donde ha sido posible analizar, su evaluación representa un gran reto para los organismos encargados de su acreditación. Se observa que uno de los principales motivos de ello se debe a los constantes cambios e innovaciones tecnológicas, pero estas no han llegado a los sistemas de evaluación; al respecto, se considera que a la par de la innovación y a los cambiantes sistemas educativos, se deberían también desarrollar reestructuraciones en las metodologías de evaluación de la calidad en la educación a distancia (García Soto, García López y Lozano Rodríguez, 2020, p. 14). 


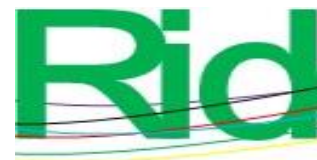

Revista Iberoamericana para la
Investigación y el Desarrollo Educativo
ISSN $2007-7467$

\section{Objetivo}

El objetivo principal de la presente investigación fue documentar el proceso seguido por la Universidad Politécnica del Valle del Évora (UPVE) para conseguir la evaluación y acreditación ante el CIEES del programa educativo licenciatura en Administración y Gestión de Pequeñas y Medianas Empresas.

\section{Metodología aplicada}

Para cumplir con el objetivo propuesto se empleó el método cualitativo, el cual permitió realizar descripciones detalladas de eventos o procesos. Este método también sirvió para revisar documentos que sustentan la creación y el desarrollo de determinada carrera, colocando el énfasis en su programa de estudios, misión, visión y modelo educativo, entre otros. Además, se realizó una investigación documental teórica de diversos autores que han escrito sobre la evaluación y la acreditación de programas de educación superior. Como el objetivo fue documentar el proceso de evaluación por los CIEES desde la gestión hasta la evaluación, se trabajó según el siguiente modelo: 


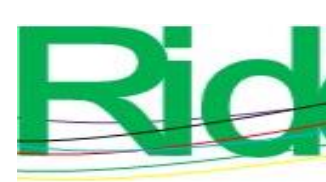

Revista Iberoamericana para la Investigación y el Desarrollo Educativo ISSN $2007-7467$

Figura 1. Modelo de evaluación a través de CIEES

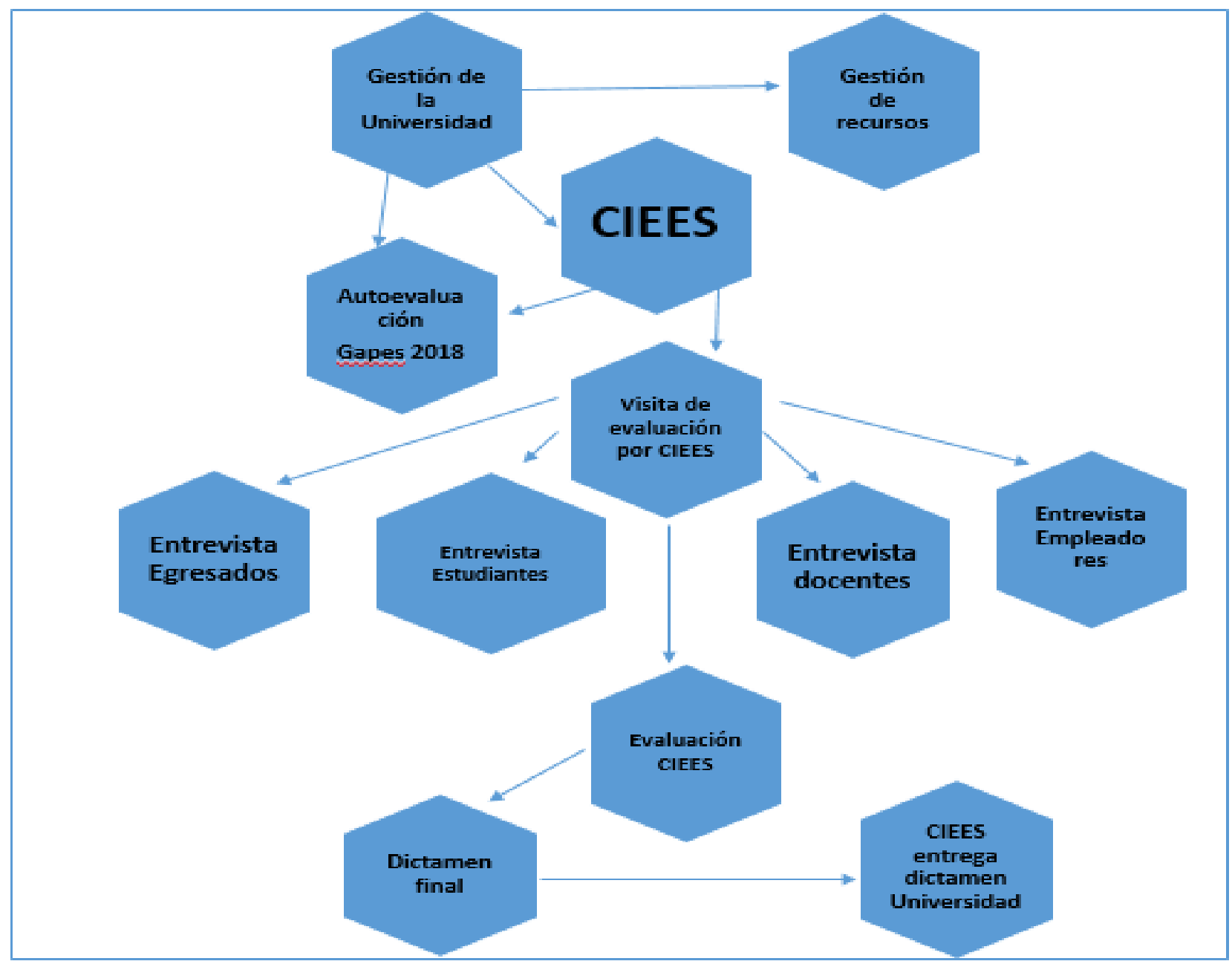

Fuente: Elaboración propia

\section{Resultados}

Luego de recibir la información necesaria por parte del CIEES sobre la acreditación de un programa educativo, el director de programas educativos de la UPVE procedió a formar equipos de trabajo conformados por tres integrantes (un profesor de tiempo completo y dos profesores del área de administración), los cuales recibieron una capacitación sobre el proceso de evaluación diagnóstica del programa educativo, así como de la plataforma GAPES (guía para la autoevaluación de programas de educación superior). Estos colaboradores se encargaron de realizar la autoevaluación diagnóstica según la información 


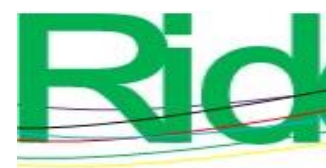

Revista Iberoamericana para la Investigación y el Desarrollo Educativo ISSN 2007 - 7467

general, los ejes y las categorías otorgadas por el CIEES como se observa en la siguiente (tabla 1).

Tabla 1. Criterios de evaluación diagnóstica según el CIEES

\begin{tabular}{|c|c|c|}
\hline Información general & Ejes & Categorías \\
\hline $\begin{array}{l}\text { Datos del programa } \\
\text { educativo. }\end{array}$ & $\begin{array}{l}\text { 1. Fundamentos y } \\
\text { condiciones de operación. }\end{array}$ & $\begin{array}{l}\text { 1. Propósito del programa. } \\
\text { 2. Condiciones generales de } \\
\text { operación del programa. }\end{array}$ \\
\hline Equipo colaborador. & $\begin{array}{l}\text { 2. Currículo específico y } \\
\text { genérico. }\end{array}$ & $\begin{array}{l}\text { 3. Modelo educativo y plan } \\
\text { de estudios. } \\
\text { 4. Actividades para la } \\
\text { formación integral. }\end{array}$ \\
\hline \multirow[t]{3}{*}{$\begin{array}{lll}\begin{array}{l}\text { Contexto del } \\
\text { educativo. }\end{array} & & \\
\text { programa } \\
\end{array}$} & $\begin{array}{l}\text { 3. Tránsito de los } \\
\text { estudiantes por el programa. }\end{array}$ & $\begin{array}{l}\text { 5. Proceso de ingreso al } \\
\text { programa. } \\
\text { 6. Trayectoria escolar. } \\
\text { 7. Egreso del programa. }\end{array}$ \\
\hline & 4. Resultados. & $\begin{array}{l}\text { 8. Resultados de los } \\
\text { estudiantes. } \\
\text { 9. Resultados del programa. }\end{array}$ \\
\hline & $\begin{array}{l}\text { 5. Personal académico, } \\
\text { infraestructura y servicios. }\end{array}$ & $\begin{array}{l}\text { 10. Personal académico. } \\
11 . \quad \text { Infraestructura } \\
\text { académica. } \\
\text { 12. Servicios de apoyo. }\end{array}$ \\
\hline
\end{tabular}

Fuente: Elaboración propia con información ofrecida por el CIEES (2019)

A continuación, se explica la información suministrada según lo referido en la tabla 1.

\section{Información general}

- Datos del programa educativo: En esta parte se refirió el nombre, el registro y el nivel académico del programa, así como el nombre de las autoridades de la institución solicitante, el total de egresados, el tipo de ciclo escolar, entre otros datos.

- Contexto del programa educativo: Se realizó una descripción precisa sobre cómo se encuentra la universidad en su entorno, el área de influencia y el acceso al municipio de Angostura (donde se ubica la universidad); en cuanto al programa educativo, se refirió el objetivo principal, a quiénes va dirigido, cuál es el campo laboral, entre otros detalles referidos al municipio (aspectos demográficos, sociales, actividades económicas, seguridad, accesibilidad, comunicación, política, etc.). 


\section{Ejes y categorías}

- Eje 1: fundamentos y condiciones de operación: Este eje estaba dividido en dos categorías:

- Categoría 1: propósitos del programa: Formar profesionistas con capacidades gerenciales altamente competitivos que respondan a los desafíos a los que se enfrentan las organizaciones en ambientes de incertidumbre, dirigiendo eficazmente sus recursos. En esta sección también se indicó que fueron aprobados por la Coordinación General de Universidades Tecnológicas y Politécnicas (CGUTyP), y se señalaron los propósitos (que incluyen investigación) y cómo se cumple con ello. Igualmente, se explicó la necesidad del programa educativo, los planes para el desarrollo y el mejoramiento del programa, así como la descripción del perfil de egreso.

- Categoría 2: condiciones generales de operación del programa: Se especificó el registro legal del programa y la normativa específica a la que está sujeto; se indicó si es difundida entre la comunidad de la universidad y si es adecuada para la operación del programa, la matrícula total y actual, así como la matrícula de ingreso en los últimos cinco años. Al respecto, se analizó críticamente si la matrícula de ingreso había aumentado o disminuido en los últimos cinco años. También se indicaron los presupuestos para el programa; sobre este aspecto, se explicó que no existe un presupuesto directo porque la universidad cuenta con un presupuesto para sus cuatro programas educativos. Por último, se señaló la estructura organizacional de la universidad y un análisis sobre si es suficiente y adecuada para la operación del programa.

- Eje 2: currículo específico y genérico: Se especificó cuál modelo educativo orientaba la creación del programa.

- Categoría 3: modelo educativo y plan de estudios: Se explicó que el modelo educativo basado en competencias sustenta la operación del programa; se explicó cómo se hace su difusión a través de conferencias dirigidas a los estudiantes y cómo es aplicado por los docentes a través de un plan de asignatura y sus evidencias correspondientes a cada unidad de la asignatura. También se describió el plan de estudios y el mapa curricular señalando las asignaturas y unidades de aprendizaje con que cuenta el programa de estudios. 


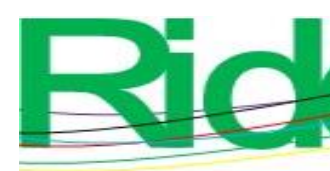

Revista Iberoamericana para la Investigación y el Desarrollo Educativo ISSN 2007 - 7467

- Categoría 4: actividades para la formación integral: Se enlistaron las siete asignaturas con valor curricular y sus unidades de aprendizaje enfocadas a la formación integral; en cuanto a la enseñanza de otras lenguas, se especificó que se tiene una asignatura de inglés en cada cuatrimestre del mapa curricular. Sobre cursos, seminarios o capacitaciones externas, se indicó que los estudiantes llevaron un curso de certificación de herramientas ofimáticas (tabla 2).

Tabla 2. Actividades para la formación integral

\begin{tabular}{|c|c|c|}
\hline Materia & Contenido temático & Objetivo \\
\hline Valores del Ser & $\begin{array}{l}\text { U1. Naturaleza humana. } \\
\text { U2. Noción y clasificación de } \\
\text { valores. } \\
\text { U3. El valor de ser persona. }\end{array}$ & $\begin{array}{l}\text { El alumno será capaz de } \\
\text { identificar y reconocer la } \\
\text { naturaleza humana, así como la } \\
\text { trascendencia de vivir los } \\
\text { valores universales en todos } \\
\text { los ámbitos del quehacer } \\
\text { humano para su propio } \\
\text { desarrollo y en función del } \\
\text { bien común. }\end{array}$ \\
\hline $\begin{array}{l}\text { Inteligencia } \\
\text { Emocional }\end{array}$ & $\begin{array}{l}\text { U1. Multidimensionalidad. } \\
\text { U2. Emoción y motivación. } \\
\text { U3. Manejo de las emociones. }\end{array}$ & $\begin{array}{l}\text { El alumno conocerá la } \\
\text { inteligencia emocional para } \\
\text { aplicarla como herramienta } \\
\text { práctica en la vida, y que le } \\
\text { permita manejar sus } \\
\text { emociones inteligentemente y } \\
\text { de esta manera elevar la } \\
\text { calidad de sus relaciones } \\
\text { consigo mismo y con los } \\
\text { demás. }\end{array}$ \\
\hline $\begin{array}{l}\text { Desarrollo } \\
\text { Interpersonal }\end{array}$ & $\begin{array}{l}\text { U1. Desarrollo interpersonal. } \\
\text { U2. Comunicación interpersonal. } \\
\text { U3. Trabajo cooperativo y } \\
\text { colaborativo. }\end{array}$ & $\begin{array}{l}\text { El alumno construirá } \\
\text { relaciones efectivas, desde la } \\
\text { comunicación y el diálogo, } \\
\text { para abrir espacios hacia una } \\
\text { auténtica humanización que } \\
\text { hace referencia a la } \\
\text { autorrealización. }\end{array}$ \\
\hline $\begin{array}{l}\text { Habilidades del } \\
\text { Pensamiento }\end{array}$ & $\begin{array}{l}\text { U1. Herramientas de pensamiento } \\
\text { concreto. } \\
\text { U2. Herramientas inferenciales. } \\
\text { U3. Pensamiento crítico. }\end{array}$ & $\begin{array}{lrr}\text { El alumno } & \text { será capaz } & \text { de } \\
\text { integrar las } & \text { herramientas } & \text { de } \\
\text { pensamiento } & \text { concreto } & \mathrm{e} \\
\text { inferencial a través del }\end{array}$ \\
\hline
\end{tabular}




\begin{tabular}{|c|c|c|}
\hline & \multicolumn{2}{|c|}{$\begin{array}{l}\text { Revista Iberoamericana para la } \\
\text { Investigación y el Desarrollo Educativo } \\
\text { ISSN } 2007-7467\end{array}$} \\
\hline & & $\begin{array}{l}\text { aprendizaje } \\
\text { descubrimiento y la práctica } \\
\text { sistemática para desarrollar } \\
\text { estructuras mentales que le } \\
\text { permitan aplicar el } \\
\text { pensamiento crítico y resolver } \\
\text { problemas de manera creativa. }\end{array}$ \\
\hline $\begin{array}{l}\text { Habilidades } \\
\text { Organizacionales }\end{array}$ & $\begin{array}{l}\text { U1. Visión organizacional. } \\
\text { U2. } \\
\text { organizacional. } \\
\text { U3. Liderazgo y trabajo en } \\
\text { equipo. }\end{array}$ & $\begin{array}{l}\text { El alumno será capaz de } \\
\text { identificar sus habilidades } \\
\text { organizacionales, tomando en } \\
\text { cuenta las fortalezas internas } \\
\text { con las que cuenta, y } \\
\text { coadyuvando al desarrollo y } \\
\text { aplicación de las mismas tanto } \\
\text { en su vida personal como en su } \\
\text { entorno. }\end{array}$ \\
\hline Ética Profesional & $\begin{array}{l}\text { U1. Generalidades de la ética. } \\
\text { U2. Implicaciones éticas en el } \\
\text { ámbito personal y profesional. } \\
\text { U3. Código ético. }\end{array}$ & $\begin{array}{l}\text { El alumno será capaz de } \\
\text { reconocer el sentido de la ética } \\
\text { comoriencia } \\
\text { especulativamente práctica del } \\
\text { obrar humano que pretende la } \\
\text { perfección y felicidad humana, } \\
\text { así como la reflexión acerca de } \\
\text { las cuestiones éticas básicas } \\
\text { del hombre en función de su } \\
\text { vida actual y de su futura vida } \\
\text { profesional. }\end{array}$ \\
\hline
\end{tabular}

Fuente: Elaboración propia con información recabada en el programa de estudios de la licenciatura en Administración y Gestión de Pequeñas y Medianas Empresas

- Eje 3: tránsito de los estudiantes por el programa: Este eje estuvo conformado por las categorías procesos de ingreso al programa, trayectoria escolar y egreso del programa.

- Categoría 5: procesos de ingreso al programa: Se presentan las estrategias de promoción y difusión del programa, las cuales se llevan a cabo a través de conferencias, carteles, trípticos, entrevistas, etc., además, se describió el procedimiento institucional de ingreso y selección al programa, así como programas de regularización y apoyo que se hacen a través del curso de inducción de tres semanas. 
- Categoría 6: trayectoria escolar: Se describió cómo opera el sistema de control escolar y estatus académico, en el que se pueden encontrar los estudiantes, la duración del plan de estudios de acuerdo con el mapa curricular, servicios de tutorías y asesorías académica, así como sus procedimientos, prácticas de estancias o visitas en el sector empleador

- Categoría 7: egreso del programa: Se presentó información sobre el proceso de titulación, requisitos, opciones de titulación, total de egresados titulados, principales obstáculos académicos y administrativos para lograr la titulación. En orientación para el tránsito a la vida profesional, se consideró información de los campos predominantes de la actividad profesional del egresado, así como la orientación que se le proporciona al estudiante para su inserción en el campo laboral, con prácticas profesionales realizadas en las asignaturas Estancia I, Estancia II y Estadía, donde el estudiante se integra a las empresas para aplicar las competencias adquiridas dentro de su carrera.

- Eje 4: resultados: Este eje estaba conformado por las categorías resultados de los estudiantes y resultados del programa.

- Categoría 8: resultados de los estudiantes: Se indicó que no se cuenta con el examen EGEL de Ceneval porque la titulación se produce de forma automática cuando se aprueban los 10 cuatrimestres del programa. En el nivel de dominio de otras lenguas, se indicó que cada uno de los nueve cuatrimestres cuenta con una asignatura de inglés. En participación de los estudiantes en cursos, competiciones, exhibiciones y presentaciones nacionales e internacionales, se aclaró que en los últimos cinco años han participado en proyectos e investigación científica, así como en congresos nacionales e internacionales donde han expuesto sus documentos de trabajo. También se realizó un análisis del cumplimiento del perfil de egreso de acuerdo con la opinión de los empleadores de la región.

- Categoría 9: resultados del programa: Se ofreció un análisis numérico de la deserción escolar del programa educativo y se señalaron las causas, así como las acciones establecidas para reducir tal índice. De igual forma, se presentó un análisis de la eficiencia terminal y eficiencia de titulación, argumentando que $100 \%$ de los egresados se encuentran con título y cédula profesional. En 


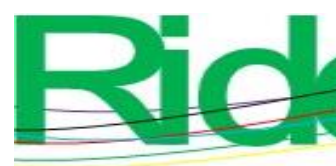

Revista Iberoamericana para la Investigación y el Desarrollo Educativo ISSN 2007 - 7467

cuanto a la empleabilidad, se indicó que se hace una encuesta de salida a los seis meses de egreso, al año y a los tres años de haber egresado para identificar la empleabilidad. Los resultados demuestran que los egresados se emplean en un periodo que oscila entre 15 días y los seis meses luego de haber obtenido el título. De igual forma, se realiza un análisis de la opinión de egresados mediante una encuesta realizada al momento de realizar sus trámites de titulación.

- Eje 5: personal académico, infraestructura y servicios: Este eje estuvo conformado por las categorías información del personal académico, infraestructura académica y servicios de apoyo.

- Categoría 10: personal académico: En este apartado se brindó información sobre el personal académico relacionada con su categoría, contratación, perfil, número de profesores, clasificados por sexo, apoyos que reciben los profesores, evaluación docente que se realiza cada cuatrimestre, superación de los profesores, actividades de docencia e investigación (se ofrecieron comprobantes de investigaciones y proyectos de los docentes presentados en congresos, publicaciones de revistas y libros).

- Categoría 11: infraestructura académica: Se describió la cantidad de equipo, aulas, laboratorios de cómputo, proyectores, auditorio y sala de maestros con que se cuentan, así como la biblioteca y su cantidad de acervo bibliográfico destinado para el programa educativo, los servicios tecnológicos (redes de internet), entre otros datos de gran importancia. Esto fue corroborado físicamente por los visitantes evaluadores de los CIEES, quienes realizaron una serie de preguntas y entrevistas al personal docente, estudiantes, egresados, empleadores, administrativos y directivos de la universidad.

- Categoría 12: Servicios de apoyo: En éste rubro se analizaron los servicios de transporte como apoyo a los estudiantes donde se indicó que la universidad cuenta con convenios de colaboración con la línea de transporte y se hace un $50 \%$ de descuento, considerando como problemático que sólo hay una línea de transporte en el municipio; así mismo la universidad cuenta con una cafetería que ofrece un menú de acuerdo a las necesidades y a las posibilidades económicas de los estudiantes y además la universidad ofrece becas de 


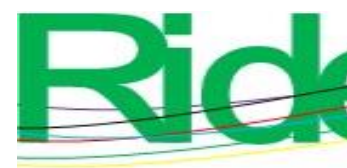

Revista Iberoamericana para la Investigación y el Desarrollo Educativo ISSN $2007-7467$

alimentos para estudiantes con problemas económicos. Todos estos servicios de apoyo fueron corroborados por los visitantes evaluadores de los CIEES, quienes además realizaron preguntas al respecto a los estudiantes seleccionados por ellos mismos para la entrevista.

\section{Discusión}

Luego de describir en el apartado anterior los pasos seguidos para el proceso de valoración de la licenciatura en Administración y Gestión de Pequeñas y Medianas Empresas, se puede indicar que el comité de los CIEES, a través de comité del área de ciencias administrativas, dictaminó la acreditación del referido programa, con algunas observaciones de mejora continua, las cuales se están solventando progresivamente para el beneficio de los aspirantes, estudiantes y egresados. Esto indica que la Universidad Politécnica del Valle del Évora cuenta con un programa de calidad avalado por un organismo externo. Este proceso, además, resulta muy útil porque permite recopilar y almacenar toda la información ordenada en ejes, categorías y subcategorías, lo que les permite a las IES tener disponible documentación concreta y fidedigna sobre un programa educativo en específico. Como limitante, sin embargo, se puede señalar que existe escasa literatura sobre el tema estudiado, en especial sobre los modelos o esquemas de evaluación empleados para la acreditación de un programa.

\section{Conclusiones}

Con base en los datos ofrecidos, se puede concluir que el trabajo en equipo de gestión y autoevaluación diagnóstica de un programa educativo resulta esencial porque permite sistematizar los datos requeridos por el organismo evaluador externo. En efecto, al realizar el proceso de evaluación y acreditación de un programa educativo, se logra integrar toda la documentación requerida, la cual abarca procesos, modelos, plan de estudios, asignaturas, filosofía, procedimientos de ingreso, egreso, titulación, tutorías, asesorías y demás información que permite a las universidades obtener un panorama más cercano de la realidad. En ese proceso, además, se consigue la participación y la opinión de estudiantes, egresados, docentes y empleadores, factores indispensables para promover mejoras en todos los aspectos analizados. En síntesis, se considera que la información suministrada en este documento 


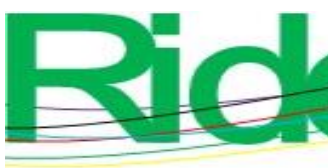

Revista Iberoamericana para la
Investigación y el Desarrollo Educativo ISSN $2007-7467$

puede servir como apoyo para otras universidades que decidan evaluar y acreditar un programa educativo.

\section{Referencias}

Acosta Ochoa, A. (2014). Evaluación y acreditación de programas educativos en México: revisar los discursos, valorar lo efectos. Revista de la Educación Superior, 4(172), Recuperado de http://publicaciones.anuies.mx/pdfs/revista/Revista172_S4A2ES.pdf Arriaga Villanueva, N. (2013). Instituciones de educación superior, beneficios de la certificación y acreditación. Revista COEPES, 3(8). Recuperado de http://www.revistacoepesgto.mx/revistacoepes8/instituciones-de-educacionsuperior-beneficios-de-la-certificacion-y-acreditacion

Buendía Espinosa, A. (2011). Evaluación y acreditación de programas en México: más allá de los juegos discursivos. Revista Diálogos sobre Educación, 2(3). Recuperado de http://dialogossobreeducacion.cucsh.udg.mx/index.php/DSE/article/view/371

Buendía Espinosa, A., Sampedro Hernández, J. L. y Acosta Ochoa, A. (2013). ¿La evaluación y acreditación mejoran la calidad de programas de licenciatura en México? La otra mirada, los estudiantes. El caso de seis universidades mexicanas. Reencuentro, $68 . \quad$ Recuperado de https://www.redalyc.org/articulo.oa?id=34030524008

Castillo Marrufo, J. A., Aragón García, M. y Hernández Jaime, J. (2014). Los procesos de acreditación; desafíos para la educación superior en México. Revista Iberoamericana de Producción Académica y Gestión Educativa, 1(1). Recuperado de https://www.pag.org.mx/index.php/PAG/article/viewFile/118/165

Comités Interinstitucionales para la Evaluación de la Educación Superior [CIESS] (2018). Proceso general para la evaluación y acreditación de programas educativos de educación superior. Recuperado de https://www.ciees.edu.mx/documentos/procesogeneral-para-la-evaluacion-y-acreditacion-de-programas-educativos-de-educacionsuperior.pdf

Consejo para la Acreditación de la Educación Superior [Copaes] (2016). Marco general de referencia para los procesos de acreditación de programas académicos de tipo $\begin{array}{llll}\text { superior } & \text { ver. } & \text { Recuperado }\end{array}$ https://www.copaes.org/documentos/Marco_de_Referencia_V_3.0_0.pdf 


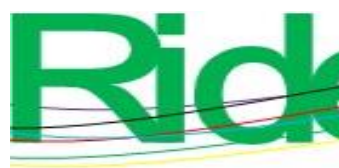

Revista Iberoamericana para la
Investigación y el Desarrollo Educativo
ISSN $2007-7467$

Corona Zapata, J. A. (2014). Programas educativos de buena calidad. Valoración de estudiantes vs expectativa de la Benemérita Universidad Autónoma de Puebla en México. Revista Actualidades Investigativas en Educación, 14(3). Recuperado de https://www.redalyc.org/pdf/447/44732048011.pdf

De la Garza Aguilar, J. (2013). La evaluación de programas educativos del nivel superior en México. Avances y perspectivas. Revista Perfiles Educativos, 35. Recuperado de http://www.scielo.org.mx/scielo.php?script=sci_arttext\&pid=S018526982013000500004

García Soto, G. Y., García López, R. I. y Lozano Rodríguez, A. (2020). Calidad en la educación superior en línea: un análisis teórico. Revista Educación, 44(2). Recuperado de https://www.redalyc.org/articulo.oa?id=44062184005

González Vidaña, B. (2014). Complejidad y sistematización de la evaluación de la educación superior en México. Revista de Comunicación de la SEECI, 33. Recuperado de https://www.redalyc.org/articulo.oa?id=523552851001

Hernández Mondragón, A. R. (2006). La acreditación y certificación en las instituciones de educación superior. Hacia la conformación de circuitos académicos de calidad: ¿Exclusión o integración? Revista Centro de Investigación Universidad la Salle, 7(26), Recuperado de https://www.redalyc.org/pdf/342/34202604.pdf

Lara Valdés, R. (2013). La acreditación y certificación de la educación superior en México: por la mejora continua. Revista COEPES, 3(8). Recuperado de http://www.revistacoepesgto.mx/revistacoepes8/la-acreditacion-y-certificacion-dela-educacion-superior-en-mexico-por-la-mejora-continua

Martínez Iñiguez, J. E., Tobón, S. y Romero Sandoval, A. (2017). Problemáticas relacionadas con la acreditación de la calidad de la educación superior en América Latina. Revista Innovación Educativa, 17(73). Recuperado de https://www.redalyc.org/pdf/1794/179450594005.pdf

Ramírez López, L. E. (2016). La evaluación de la educación superior: un campo de tensiones. Revista de Difusión Cultural y Científica de la Universidad La Salle, 11(11). Recuperado de www.scielo.org.bo/scielo.php?script=sci_arttext\&pid=S2071081X2016000100009

Rodríguez Pérez, I. (2014). La acreditación de los programas educativos. ¿Eleva la calidad de educación? Revista Iberoamericana de Producción Académica y Gestión 


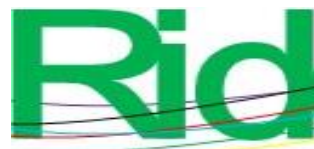

Educativa,

https://pag.org.mx/index.php/PAG/article/viewFile/198/246

Rosales Almazán, I. (2019). La evaluación en nivel superior. Revista Atlante: Cuaderno de $\begin{array}{lllll}\text { Educación } & y & \text { Desarrollo. } & \text { Recuperado de }\end{array}$ https://www.eumed.net/rev/atlante/2019/02/evaluacion-nivel-superior.html

Rubio Oca, J. (2007). La evaluación y acreditación de la educación superior en México: un largo camino aún por recorrer. Revista Reencuentro, 50. Recuperado de https://www.redalyc.org/pdf/340/34005006.pdf

Rueda Beltrán, M. y García Salord, S. (2013). La evaluación en el campo de la educación superior. Revista Perfiles Educativos, 35. Recuperado de http://www.scielo.org.mx/scielo.php?script=sci_arttext\&pid=S018526982013000500002

Zenteno Trejo, B. Y., Osorno Sánchez, A. y López Portillo Tostado, V. (2017). El consejo para la acreditación de la educación superior COPAES en México: retos y reflexiones. Revista de Educación y Derecho, 15. Recuperado de https://revistes.ub.edu/index.php/RED/article/view/18319/20949 